\title{
Is Kirschner Wire (K-wire) Infection Risk Higher when Operated by Junior Surgeons?-Infection Rates of K-wire Fixation in a UK Plastic Surgery Hand Trauma Unit
}

\author{
Martha Fatima Irene De La Cruz Monroy ${ }^{1,2, \odot ~} \quad$ Zabihullah Abdul $^{1} \quad$ Kameel Khan $^{1} \quad$ Zakir Shariff $^{1}$
}

\author{
1Plastic Surgery Department, Bradford Royal Infirmary, Bradford \\ Teaching Hospitals Foundation Trust, Bradford, United Kingdom \\ 2Plastic Surgery Department, Sheffield Teaching Hospitals NHS \\ Trust, Sheffield, United Kingdom
}

Indian J Plast Surg 2021;54:235-236.

Hand injuries such as fractures and dislocations are commonly managed with K-wires. Studies assessing K-wire complications suggest an infection rate between $6.6 \%$ to $33 \%{ }^{1-5}$ Our local hand trauma unit perceived an increase in frequency in K-wire infections. Therefore, we assessed our local hand injury K-wire infection rate and its associated factors.

The primary outcome was to identify the infection rate while the secondary outcome was to compare injury, surgical and postoperative factors of cases which developed infections compared with those which did not. This retrospective analysis included all cases (adult and pediatric) requiring at least one K-wire fixation in the plastic surgery trauma theater lists between August and December 2019. Data collected included the following: patient demographics, injury characteristics, surgery characteristics, timing of appointments, and follow-up.

We identified 54 cases: 46 noninfected and 8 infected, leading to an infection rate of $15 \%$ ( - Fig. 1 a). Differences between demographics ( - Fig. $\mathbf{1}$ b), injury characteristics and surgical characteristics such as K-wire number, antibiotic prophylaxis and follow-up were not statistically significant. Interestingly, $59 \%$ of noninfected cases and $100 \%$ of infected cases were operated by junior surgeons (registrars), and this difference was statistically significant $(p=0.015$, -Fig. 1 c); however, the registrar grade (junior vs. senior registrar) difference was not $(p=1)$. Additionally, we noted that differences in the degree of involvement of the senior supervisor (consultant) was statistically significant $(p=0.0007$,

-Fig. $1 \mathbf{d}$ ). For a summary of the results and their statistical analyses, please see $\boldsymbol{-}$ Table $\mathbf{1}$.
Address for correspondence Martha F. I. De La Cruz Monroy, BSc (Hons), MB, ChB, MRCS (Eng), MSc, Plastic Surgery Department, Bradford Royal Infirmary, Duckworth Lane, Bradford, BD9 6RJ United Kingdom (e-mail: martha.delacruzmonroy@nhs.net).

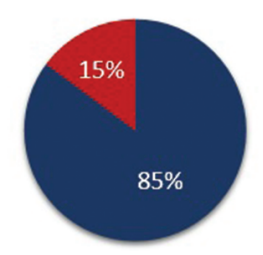

A - Non-infected Infected

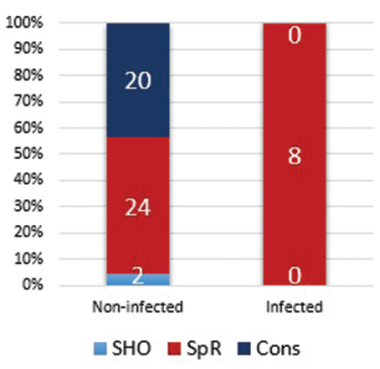

C

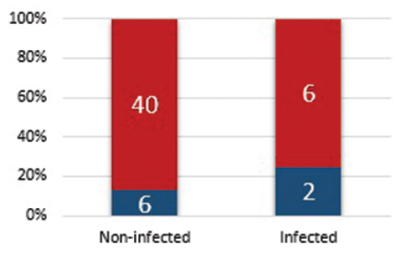

B

a Female Male

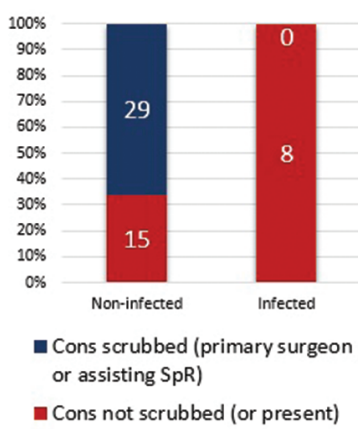

Fig. 1 (a) Graph showing the infection rate. (b) Graph showing patient demographics ( $p=0.38$, difference is nonstatistically significant, Fisher's exact test). (c) Graph showing the difference in frequency of surgeon level per group $(p=0.015$, difference is statistically significant, Fisher's exact test). (d) Graph showing the differences in level of consultant involvement per group ( $p=0.0007$, difference is statistically significant, Fisher's exact test. (Statistical analyses performed through JASP Version 0.13.1 Computer Software). Key: Cons: Consultant, SpR: registrar, SHO: senior house officer. published online March 4, 2021
DOI https://doi.org/

$10.1055 / \mathrm{s}-0041-1723906$ ISSN 0970-0358. (c) 2021. Association of Plastic Surgeons of India.

This is an open access article published by Thieme under the terms of the Creative Commons Attribution-NonDerivative-NonCommercial-License, permitting copying and reproduction so long as the original work is given appropriate credit. Contents may not be used for commercial purposes, or adapted, remixed, transformed or built upon. (https://creativecommons.org/licenses/by-nc-nd/4.0/).

Thieme Medical and Scientific Publishers Pvt. Ltd. A-12, 2nd Floor, Sector 2, Noida-201301 UP, India 
Table 1 Summary of results and factors analyzed while assessing differences between the noninfected and infected hand injury K-wire cases. (Statistical analyses performed through JASP Version 0.13.1 Computer Software)

\begin{tabular}{|c|c|c|c|}
\hline & $\begin{array}{l}\text { Noninfected } \\
(n=46)\end{array}$ & $\begin{array}{l}\text { Infected } \\
(n=8)\end{array}$ & $\begin{array}{l}p \text {-value } \\
(95 \% \mathrm{Cl})\end{array}$ \\
\hline \multicolumn{4}{|l|}{ Demographics } \\
\hline $\begin{array}{l}\text { Age per patient group } \\
\text { (years) }\end{array}$ & 34 & 37 & 0.672 \\
\hline Female & $6 / 46$ & $2 / 8$ & 0.38 \\
\hline \multicolumn{4}{|l|}{ Injury factors } \\
\hline $\begin{array}{l}\text { Cases across the months } \\
\text { (Aug-Dec 2019) }\end{array}$ & \multicolumn{2}{|l|}{-} & 0.26 \\
\hline $\begin{array}{l}\text { Injury anatomical location } \\
\text { (specific digit) }\end{array}$ & \multicolumn{2}{|l|}{-} & 0.402 \\
\hline Injuries per hand (1 vs. $2+$ ) & \multicolumn{2}{|l|}{-} & 0.474 \\
\hline $\begin{array}{l}\text { Open injuries (vs. closed } \\
\text { injuries) }\end{array}$ & $6 / 46$ & $0 / 8$ & 0.279 \\
\hline \multicolumn{4}{|l|}{ Surgery factors } \\
\hline $\begin{array}{l}\text { Days between } 1 \text { st assess- } \\
\text { ment and surgery }\end{array}$ & 1.1 & 2.2 & 0.241 \\
\hline $\begin{array}{l}\text { SpR as primary surgeon (vs. } \\
\text { consultant) }\end{array}$ & $24 / 44^{a}$ & $8 / 8$ & $0.015^{\mathrm{a}}$ \\
\hline $\begin{array}{l}\text { Junior SpR (vs Senior SpR, if } \\
\text { SpR primary surgeon) }\end{array}$ & $13 / 24$ & $5 / 8$ & 0.681 \\
\hline $\begin{array}{l}\text { Consultant absent (vs. pres- } \\
\text { ent scrubbed/unscrubbed, if } \\
\text { SpR primary surgeon) }\end{array}$ & $12 / 24$ & $7 / 8$ & 0.061 \\
\hline $\begin{array}{l}\text { Consultant not scrubbed } \\
\text { (vs. scrubbed, if SpR primary } \\
\text { surgeon and consultant in } \\
\text { theater) }\end{array}$ & $3 / 12$ & $1 / 1$ & 0.118 \\
\hline $\begin{array}{l}\text { Consultant not scrubbed/ } \\
\text { absent (vs. consultant } \\
\text { scrubbed as primary sur- } \\
\text { geon or supervising) }\end{array}$ & $15 / 44^{\mathrm{a}}$ & $8 / 8$ & $<0.001^{a}$ \\
\hline $\begin{array}{l}\text { Skin preparation chlorhex- } \\
\text { idine (vs. Povidone iodine } \\
\text { based or NS) }\end{array}$ & $22 / 46$ & $6 / 8$ & 0.279 \\
\hline 1 K-wire (vs 2 or more) & $15 / 46$ & $3 / 8$ & 0.786 \\
\hline \multicolumn{4}{|l|}{ Antibiotic prophylaxis $(\mathrm{n}=54)$} \\
\hline $\begin{array}{l}\text { Prophylactic given on induc- } \\
\text { tion (vs. not given/NS) }\end{array}$ & $24 / 46$ & $6 / 8$ & 0.125 \\
\hline $\begin{array}{l}\text { Prophylactic on discharge } \\
\text { (vs. not given/NS) }\end{array}$ & $11 / 46$ & $0 / 8$ & 0.121 \\
\hline \multicolumn{4}{|l|}{ Follow-up } \\
\hline $\begin{array}{l}\text { 1st pin site check (days } \\
\text { postop) }(n=54)\end{array}$ & 10.8 & 8.7 & 0.761 \\
\hline $\begin{array}{l}\text { Time K-wires in situ (days) } \\
(n=53)^{b}\end{array}$ & 27.7 & 20.8 & $0.0083^{a}$ \\
\hline
\end{tabular}

Abbreviations: $\mathrm{Cl}$, confidence interval; NS, not specified; SpR, registrar. a 2 cases excluded as they were operated by senior house officers and supervised by registrars. ${ }^{\mathrm{b}} 1$ case excluded as followed-up in a different city.
Although no studies comparing skills or outcomes of junior versus senior surgeons have been identified, surgical technique has been highlighted as an important factor in determining pin site infection in orthopedic limb surgery. ${ }^{6}$ For example, infection risk reducing technique skills (such as nontouch handling of wires and minimizing hematoma on the surgical site) are likely to improve with experience and practice. These may not have been developed by the registrars in this study, which could have led to an increased infection rate.

In conclusion, we report an infection rate of $15 \%$ and note that most surgical and injury factors' differences between infected and noninfected groups were nonsignificant. We note a significance in the level of primary surgeon and consultants' involvement with the case. We recommend that further consultant supervision is implemented when registrars are performing the operations as well as further training and education. A larger study to assess these measures' effectiveness in the reduction of infection rates is required.

\section{Conflicts of Interest}

None declared.

\section{References}

1 Hsu LP, Schwartz EG, Kalainov DM, Chen F, Makowiec RL. Complications of K-wire fixation in procedures involving the hand and wrist. J Hand Surg Am 2011;36(4):610-616

2 McGonagle L, Elamin S, Wright DM. Buried or unburied K-wires for lateral condyle elbow fractures. Ann R Coll Surg Engl 2012;94(7):513-516

3 Terndrup M, Jensen T, Kring S, Lindberg-Larsen M. Should we bury K-wires after metacarpal and phalangeal fracture osteosynthesis? Injury 2018;49(6):1126-1130

4 Gardiner MD, Gardiner S, Issa F, et al; WIRE Research Collaborative. Buried versus exposed kirschner wires following fixation of hand fractures: L Clinician and patient surveys. Plast Reconstr Surg Glob Open 2018;6(4):e1747

5 Wormald JCR, Jain A, Lloyd-Hughes H, Gardiner S, Gardiner MD. A systematic review of the influence of burying or not burying Kirschner wires on infection rates following fixation of upper extremity fractures. J Plast Reconstr Aesthet Surg 2017;70(9):1298-1301

6 Davies R, Holt N, Nayagam S. The care of pin sites with external fixation. J Bone Joint Surg Br 2005;87(5):716-719 